\title{
EL “COCODRILO DE TUMBES” (Crocodylus acutus Cuvier 1807): ESTUDIO PRELIMINAR DE SU ESTADO ACTUAL EN EL NORTE DE PERÚ.
}

\author{
Armando H. Escobedo Galván ${ }^{1}$ y Fernando Mejía Vargas $^{2}$
}

\section{Resumen:}

De Junio del 2002 a Febrero del 2003, se han realizado recorridos en la zona norte de Perú, específicamente en la región de Tumbes, para conocer el estado actual de Crocodylus acutus, mediante recorridos diurnos y nocturnos, estimando una densidad de $0.18 \mathrm{ind} / \mathrm{Km}$ siendo una de las densidades más bajas de C. acutus en su ámbito de distribución. La estructura poblacional es de $82.61 \%$ juveniles, $4.35 \%$ de sub-adultos y $13.04 \%$ de adultos, siendo similar a lo reportado en otras poblaciones.

Palabras claves: Crocodylus acutus, Cocodrilo de Tumbes, estructura poblacional, Río Tumbes.

\begin{abstract}
In order to assess the actual status of the Crocodylus acutus populations from northern Perú, Tumbes Region, from June 2002 to February 2003, diurnal and nocturnal surveys were conducted. A density of 0.19 indviduals / $\mathrm{km}$ was estimated. This is the lowest density for C. acutus worldwide. The following population structure was found: $82.61 \%$ juveniles, $4.37 \%$ subadults and $13.09 \%$ adults. This is similar to population structures reported elsewhere.
\end{abstract}

Key words Crocodylus acutus, Crocodile of Tumbes, it structures populational, Tumbes River.

\section{Introducción}

Crocodylus acutus, conocido como "Lagarto", "Cocodrilo Americano" o "Cocodrilo de Tumbes", está presente en ambas costas de la región neotropical del continente americano, desde el extremo sur de Florida, hasta los llanos del Orinoco en el noreste de Venezuela, en el Atlántico, mientras que en el Pacífico va desde Sinaloa en México, hasta el norte de Perú (Medem, 1983; Thorbjarnarson, 1989).

A pesar de su amplia distribución, sus poblaciones han sufrido una disminución debido principalmente a la pérdida de sus hábitats y la cacería furtiva. Esto ha hecho, que se encuentre en peligro de extinción (Thorbjarnarson, 1988; Ross, 1998).

Por ello, el Cocodrilo de Tumbes, ha sido incluido dentro del Apéndice I del Convenio Internacional para el Tráfico de Especies Amenazadas de Flora y Fauna (CITES, 1995), como consecuencia dentro de su ámbito de distribución se le considera una especie en peligro de extinción que debe ser protegida, razón por la cual no se permite la comercialización de productos y subproductos de esta especie provenientes de su hábitat silvestre. Además, en Perú, desde 1950, bajo la Resolución Suprema N ${ }^{\circ}$ 345, se prohíbe la caza de Crocodylus acutus en su hábitat natural.
A pesar de las leyes vigentes tanto internacionales como nacionales para la conservación del "Cocodrilo de Tumbes", su situación no se ha revertido como debería de esperarse.

En Perú, esta especie tiene una distribución histórica que abarcaba desde el río Zarumilla por el norte hasta en río Chira por el sur (Medem, 1983), sin embargo las últimas evaluaciones han reportado una aparente desaparición de esta especie en las cuencas del Río Chira.

\section{Antecedentes}

El primer censo de C. acutus, para conocer su situación en la región, se llevó acabo en 1970, registrando un total de 40 individuos para toda la cuenca del Río Tumbes, posteriormente en 1980, solo se registró 5 individuos en un sector del Río Tumbes, para 1985, se registraron cuatro ejemplares en $12 \mathrm{Km}$. del estero corrales en los manglares de Tumbes (Vásquez \& Pickens, 1995) y recientemente, en 1997, miembros del Grupo de Especialistas de Cocodrilos determinaron la presencia de no más de 6 parejas reproductivas en la zona.

El objetivo del presente trabajo fue determinar el estado actual de la población de Crocodylus acutus en la zona de Tumbes.

\footnotetext{
${ }^{1}$ Escuela de Ciencias Ambientales, Universidad Latina de Costa Rica y Asociación Costarricense de Investigadores en Crocodilidos. Apartado Postal: 27-2100 Guadalupe, San José, Costa Rica.

Correo electrónico: elchorvis@yahoo.com

${ }^{2}$ Universidad San Luis Gonzaga de Ica. Av. San Martín 465 la Tinguiña Ica-Perú. Correo electrónico: fermejia67@hotmail.com
} 


\section{Material y Métodos:}

El estudio se realizó desde Junio del 2002 a Febrero del 2003. La evaluación se llevó acabo en el Río Tumbes (límites del Parque Nacional Cerros de Amotape y la Zona Reservada de Tumbes) desde el sector denominado Cóndor Flores hasta el sector Bocana Carrillo incluyendo los sectores de Bocana Murciélago, Cabo Inga y el Guanábano. También se evaluaron los esteros conocidos como La Chepa, Barranco Blanco y La Canela.

Se utilizó la metodología descrita por Chabreck (1966), que consiste en el conteo visual nocturno, ya que los ojos de los cocodrilos poseen un tapetum lucidum, refractor de luz, el cual presenta brillo de los ojos fueron designados como "ojos" (King et al., 1990).

\section{Resultados y Discusión:}

Se realizaron 15 recorridos (2 recorridos por sector, excepto el de el estero la Canela), para un total de $121.5 \mathrm{Km}$, observándose 22 individuos. Se estimó una densidad de $0.18 \mathrm{ind} / \mathrm{Km}$, siendo una de las densidades más bajas para la especie en todo su rango de distribución, este valor puede verse afectado por factores climáticos (principalmente el fenómeno de El Niño y La Niña), la estación, el efecto de la marea y la hora en que se realizó el estudio (Cerrato, 1991), además de ser la zona de

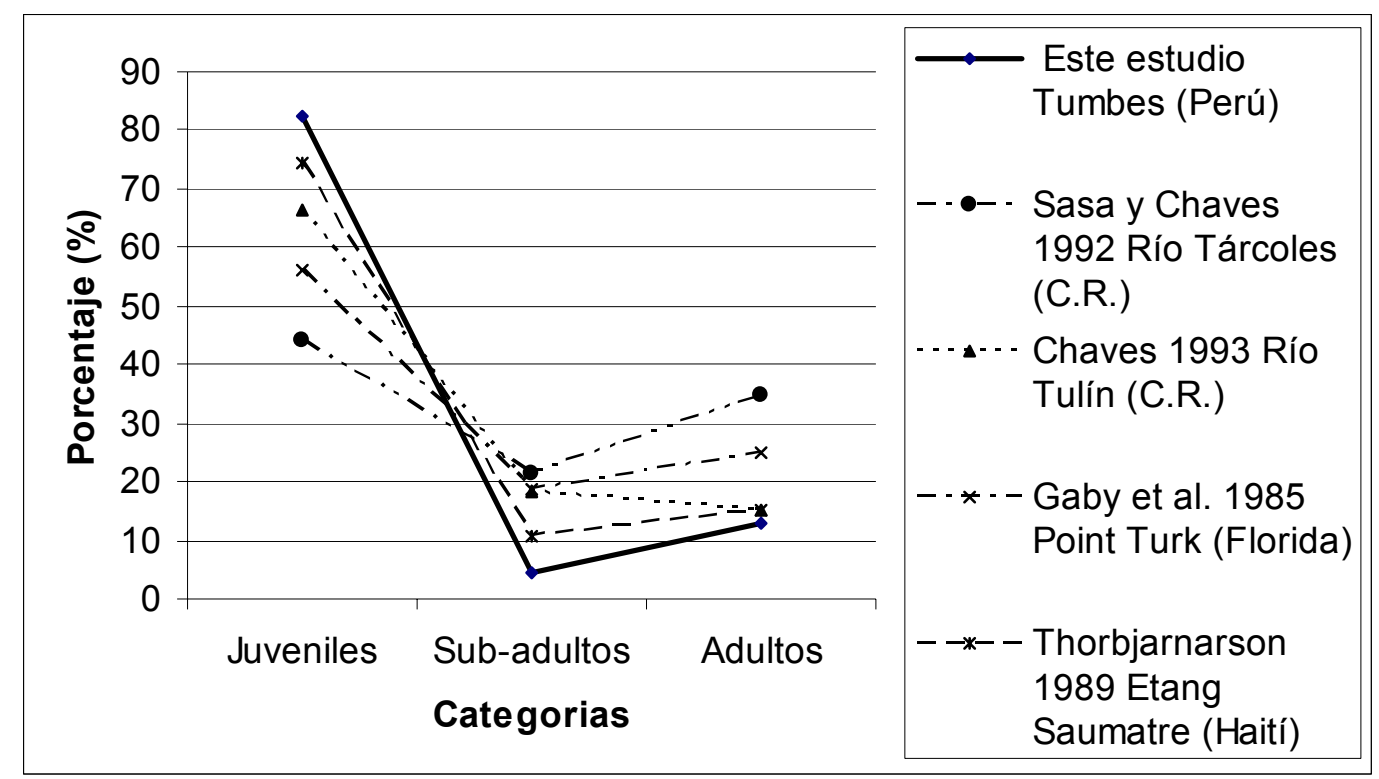

Figura 1. Estructura y tendencia de la población de C. acutus estimada para la zona de Tumbes, en comparación con otras poblaciones estimadas de la especie.

un color rojizo que permite detectarlos hasta una distancia de 200 metros cuando se les alumbra en la noche (Salas, 1985).

Para evaluar la población de esta especie se realizó primero un reconocimiento del área de estudio en el día y luego observaciones directas diurnas y nocturnas en las zonas antes determinadas, en el sector del Río Tumbes, específicamente en el sector denominados El Guanabano, Bocana Carrillo y Quebrada Honda. Los especímenes se estudiaron desde la orilla del río, en el sector de los esteros de manglares y también en forma directa en bote o canoas.

Se calculó el tamaño de los individuos observados durante los recorridos, mediante la estimación de la longitud cefálica y distancia interocular. Las estimaciones fueron agrupadas en tres categorías, basadas en la clasificación de Thorbjarnarson (1989): a) juveniles $<90 \mathrm{~cm}$, b) Sub-adultos de 90 a $180 \mathrm{~cm}$, y c) Adultos $>$ de 180 $\mathrm{cm}$. Aquellos individuos que sólo mostraron
Tumbes el límite sur de distribución, no es precisamente una especie abundante ya que enfrenta la problemática de ser una población limítrofe, al no estar en contacto con otras poblaciones (se desconoce el estado de C. acutus en Ecuador). Ello conduce a que no se vea beneficiada por la recolonización conforme se pierden individuos locales, además de reducir depresiones poblacionales debido a la consanguinidad.

La expansión de las diferentes actividades antrópicas en las márgenes del Río Tumbes y los esteros como, la actividad ganadera, expansión de tierras agrícolas y actividades ilegales como la extracción de la madera, la caza furtiva de la fauna silvestre y la extracción de oro en forma artesanal traen como consecuencia la presencia de personas en forma permanente en zonas que antes fueron el hábitat de esta especie quedando sin zonas de reproducción y como consecuencia, se refugian en zonas cada vez más inaccesibles para el hombre. 
En el caso de los esteros la expansión agrícola y la actividad langostinera ha crecido de tal manera que en los sectores del estero la Chepa y Barranco Blanco estas expansiones llegan hasta la orilla de los esteros, y debido a esta expansión se ha talado casi la totalidad del manglar. En algunos casos, los cocodrilos son cazados o capturados muchas veces para ser comercializados vivos en la zona de Puerto Pizarro.

La estructura poblacional en la zona estudiada, presenta un gran cantidad de juveniles, los cuales representan un $82.61 \%$, mientras que los subadultos y adultos representan un $4.35 \%$ y $13.04 \%$ respectivamente. Este tipo de estructura poblacional a sido observada en otros estudios (Thorbjarnarson, 1989; Sasa \& Chaves, 1992; Piedra, 2000), la cual puede estar asociada al tipo de estrategia poblacional de la especie para su sobre vivencia (Figura 1).

El bajo porcentaje de sub-adultos puede deberse a dos causas; a que esta clase es muy difícil de observar, lo cual genera un sesgo en los muestreos y que la etapa de sub-adulto es relativamente corta, llegando a la madurez sexual en poco tiempo (Sasa \& Chaves, 1992).

Sin embargo, la evaluación de C. acutus, presentada en este trabajo no constituye un censo poblacional completo, sino una indicación del número mínimo de cocodrilos que se encuentran en la zona.

\section{Agradecimientos:}

Agradecemos a Luis Escobedo Cantú, Socorro Galván Carreón, por su apoyo para la realización de este estudio, a Carlos Arias por su hospitalidad y aporte logístico, al personal del Instituto Nacional de Recursos Naturales (INRENA) en Tumbes especialmente a Fernando Cuadros por su ayuda en la realización del estudio, a Karina Ramírez, Pavel Cartagena y a la Lic. Rosario Acero directora del INRENA por su apoyo en cuanto a los permisos, y por último un agradecimiento especial a la Dra. Miryam Venegas de Anaya por el aporte con su valioso conocimiento.

\section{Literatura citada:}

Cerrato C. 1991. Composición y Tamaño de Poblaciones Silvestres de Caimanes (Caiman crocodilus chiapasus) y Cocodrilos (Crocodylus acutus) de la Costa Caribe de Honduras, Centro América. Tesis de Maestría para optar al grado de Magister Sceientiae en Manejo y Conservación de Vida Silvestre. Programa Regional en Manejo de Vida Silvestre para Mesoamérica y el Caribe. Universidad Nacional. Heredia, Costa Rica.
Chabreck R.H. 1966. Methods of determining the size and composition of Alligátor population in Luisiana. Proc. $20^{\text {th }}$ Ann. Conf. S.E. Assoc. Game Fish Comm. 20: 105-112.

Chaves G. 1993. Situación poblacional del cocodrilo amarillo (Crocodylus acutus Reptilia, Crocodilidae) en el Pacifico Central de Costa Rica. Ecología. Reporte científico $N^{o}$ 2. Universidad Estatal a Distancia.

Convención Internacional para el Tráfico de Especies Amenazadas de Flora y Fauna Silvestre (CITES). 1995. Convención para el Comercio de Especies Amenazadas. U.S. Departament of Interior. Fish and Wildlife Service. Washington.

King F.W., Espinal M. \& Cerrato C. 1990. Distribution and status of the crocodilians of Honduras. Results of a survey conducted for the convention on International Trade in Endangered Species of Wild Fauna and Flora and the Honduras Secretaría de Recursos Naturales.

Medem F. 1983. Los Crocodylia de Colombia. Vol. II. Editorial Carrera. Bogotá, Colombia.

Piedra L. 2000. Estado de las poblaciones de cocodrilos (Crocodylus acutus) (Reptilia: Crocodylidae) en tres ríos del Pacífico Central de Costa Rica. Tesis de Licenciatura. Universidad Nacional de Costa Rica.

Ross J.P. 1998. Crocodiles. Status survery and conservation action plan. $2^{\text {nd }}$ Edition. UICN/SSC. Crocodile Specialist Group. Gland, Switzerland and Cambridge, UK. Viii.

Salas C.E. 1985. Contribución al conocimiento sobre el manejo del Crocodylus acutus Cuvier (Crocodylia, Crocodylidae) en el Refugio Nacional de Fauna Silvestre Dr. Rafael Lucas Rodríguez Caballero. Universidad de Costa Rica, San José, Costa Rica.

Sasa M. \& Chaves G. 1992. Tamaño, estructura y distribución de una población de Crocodylus acutus (Crocodylia: Crocodylidae) en Costa Rica. Rev. Biol. Trop. (40): 131-134.

Thorbjarnarson J.B. 1988. Status and ecology of American crocodile in Haiti. Bull. Fla. State Museum. 33(1): 1-86.

Thorbjarnarson J.B. 1989. Ecology of the American Crocodyle, Crocodylus acutus, in Crocodiles international Union for the conservation of the Nature, Gland, Switzerland. : 228-257.

Vásquez P.G. \& Pickens C. 1995. Estado de Conservación de los Crocodylia en el Perú. pp. 135-157. En: Larriera A. \& Verdade L. (Eds.) La Conservación y el Manejo de caimanes y cocodrilos de América Latina, Vol. 1. Fundación Banco Bica. Santo Tomé, Santa Fe, Argentina. ISBN-950-9632-21-X. 